Astrophysics and Space Science manuscript No.

(will be inserted by the editor)

Diego F. Torres et al.

\title{
INTEGRAL/XMM views on the MeV source GRO J1411-64
}

Received: date / Accepted: date

\begin{abstract}
The COMPTEL unidentified source GRO J 1411-64 was observed by INTEGRAL and XMM-Newton in 2005. The Circinus Galaxy is the only source detected within the $4 \sigma$ location error of GRO J1411-64, but in here excluded as the possible counterpart. At soft X-rays, 22 reliable and statistically significant sources (likelihood $>10$ ) were extracted and analyzed from XMM-Newton data. Only one of these sources, XMMU J141255.6 635932 , is spectrally compatible with GRO J1411-64 although the fact the soft X-ray observations do not cover the full extent of the COMPTEL source position uncertainty make an association hard to quantify and thus
\end{abstract}

Diego F. Torres

Institució de Recerca i Estudis Avançats (ICREA) \& Institut de Ciències de l'Espai (IEEC-CSIC), Facultat de Ciencies, Universitat Autònoma de Barcelona, Torre C5 Parell, 2a planta, 08193 Barcelona, Spain E-mail: dtorres@ieec.uab.es

Shu Zhang

Laboratory for Particle Astrophysics, Institute of High Energy Physics, Beijing 100049, China

Olaf Reimer

W. W. Hansen Experimental Physics Laboratory, Stanford University, Stanford, CA 94305, USA

Xavier Barcons, Amalia Corral

Instituto de Física de Cantabria (CSIC-UC), E-39005 Santander, Spain

Valentí Bosch-Ramon, Josep M. Paredes

Universitat de Barcelona, Av. Diagonal 647, E08028, Barcelona, Spain

Gustavo E. Romero

Instituto Argentino de Radioastronomia, CC5, 1894, Villa Elisa, Argentina

Jin $\mathrm{Lu} \mathrm{Qu}$

Laboratory for Particle Astrophysics, Institute of High Energy Physics, Beijing 100049, China

Werner Collmar, Volker Schönfelder

Max-Planck-Institut für extraterrestrische Physik, PO Box 1603, D-85740 Garching, Germany

Yousaf Butt

Harvard-Smithsonian Center for Astrophysics, 60 Garden St., Cambridge, MA 02138, USA risky. At the best location of the source, detections at hard X-rays show only upper limits, which, together with $\mathrm{MeV}$ results obtained by COMPTEL suggest the existence of a peak in power output located somewhere between $300-700 \mathrm{keV}$ for the so-called low state. Such a spectrum resembles those in blazars or microquasars, and might suggest at work by a similar scenario. However, an analysis using a microquasar model consisting on a magnetized conical jet filled with relativistic electrons, shows that it is hard to comply with all observational constrains. This fact and the non-detection at hard Xrays introduce an a-posteriori question mark upon the physical reality of this source, what is discussed here.

Keywords $\gamma$-rays $\cdot$ unidentified $\gamma$-ray sources

\section{PACS}

\section{Introduction}

GRO J1411-64 is the strongest variable unidentified MeV source located near the Galactic plane. It was dicovered by COMPTEL/CGRO during 1995 March-July (viewing periods 414-424), during which the source went on a burst event at $\mathrm{MeV}$ energies (Zhang et al. 2002). The source was detected at $\sim 7 \sigma$ in the $1-3 \mathrm{MeV}$ band by combining the 7 viewing periods (VPs, the periods of observations in CGRO), according to which the best location was measured at $(\mathrm{l}, \mathrm{b})=\left(311.5^{\circ},-2.5^{\circ}\right)$ and the source was referred as GRO J1411-64. The flare duration was several months and the rather steep spectral shape obtained while the source was flaring would predict a bright, hard X-ray source, if there is no break in the spectrum, which is explored here. In what follows, we present the results of the INTEGRAL observations of this source, as well as of XMM-Newton observation of its best location and, followingly, the comment concerning the possible nature of this source. 


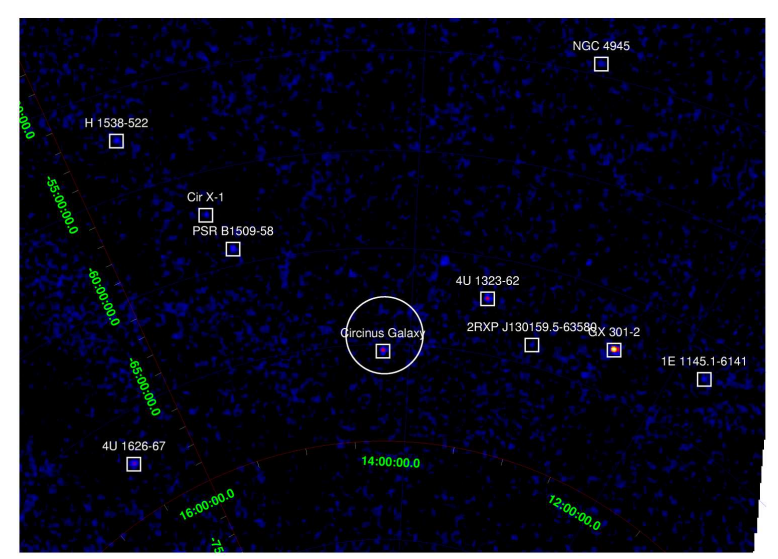

Fig. 1 Sky map of the GRO J1411-64 region as seen by IBIS/ISGRI in the $20-40 \mathrm{keV}$ range, by combining all data obtained in the observations performed during 2004 December 30 to 2005 January 6.

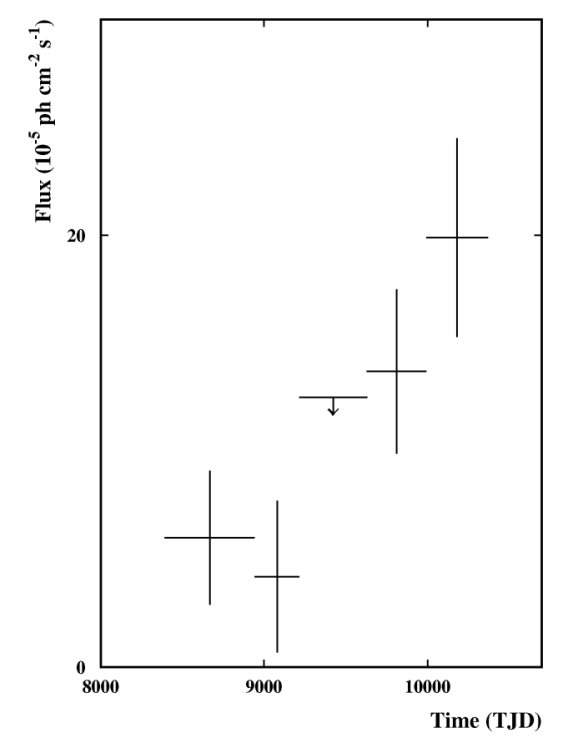

Fig. 2 Light curve of GRO J1411-64 as observed by COMPTEL at $0.75-1 \mathrm{MeV}$ band. The error bar is $1 \sigma$ and upper limit $2 \sigma$. These data points include the 7 viewing periods when the source was flaring.

\section{Observation and Data Analysis}

GRO J1411-64 was observed by INTEGRAL during 2004 December 30 - 2005 January 6. In total, 102 science windows (scws) were carried out to have $210 \mathrm{ks}$ of effective exposure. Data reduction was performed using the version 5.0 of the standard Offline Science Analysis (OSA) software, and the spectra were fitted with XSPEC of FTOOLS 5.3.1.

The best localization of COMPTEL source GRO J141164 was observed with XMM-Newton during revolution 960 on the 7th of March of 2005 (Obs. ID: 0204010101). The data were pipeline-processed with the XMM-Newton Science Analysis Software (SAS) version 6.1. After re-

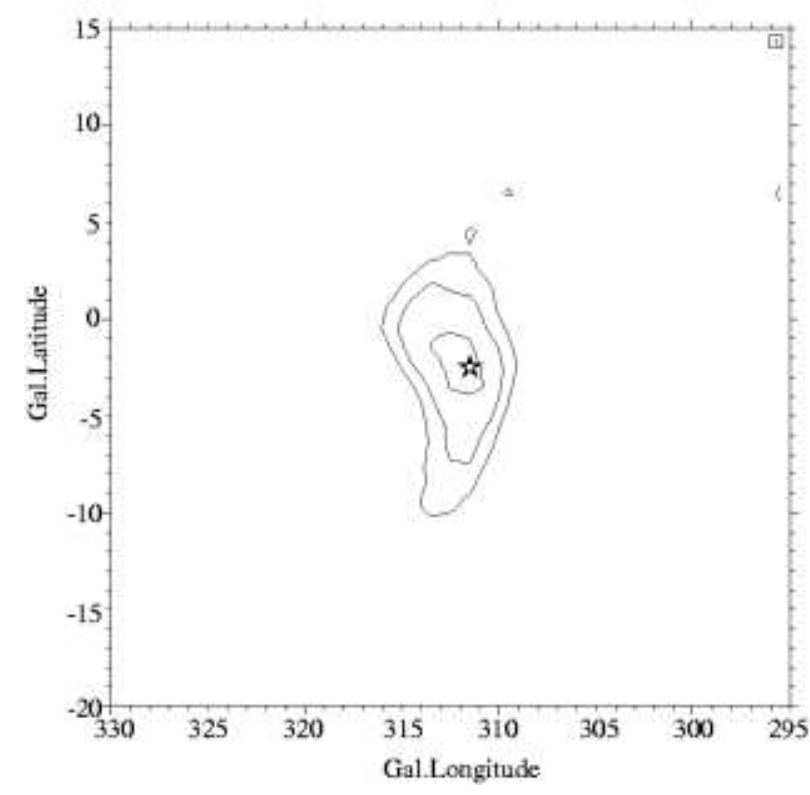

Fig. 3 The skymap of GRO J1411-64 as observed by COMPTEL in 0.75-1 MeV during 1991-1996, not including the flare period of 4 months in 1995. The star represents the bestguessed source location. The contour lines start at a detection significance level of $3 \sigma$ with steps of $0.5 \sigma$.

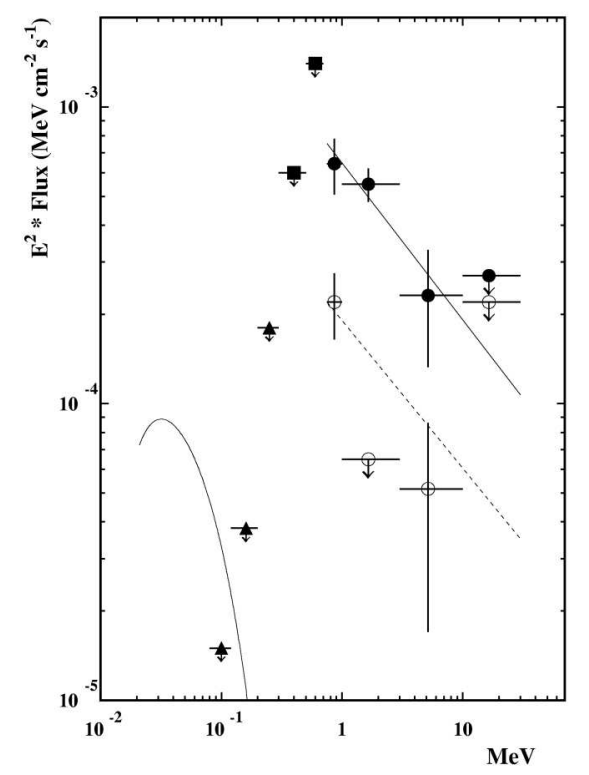

Fig. 4 Combined energy spectrum of GRO J1411-64. Filled (open) circles represent flare (low) states at $\mathrm{MeV}$ energies, solid line for the flare state, dashed line for the low state, and the $2 \sigma$ upper limits obtained from IBIS/ISGRI (triangles) and SPI (squares). The solid curve at low energies is the energy spectrum of Circinus Galaxy derived from the IBIS/ISGRI data. 


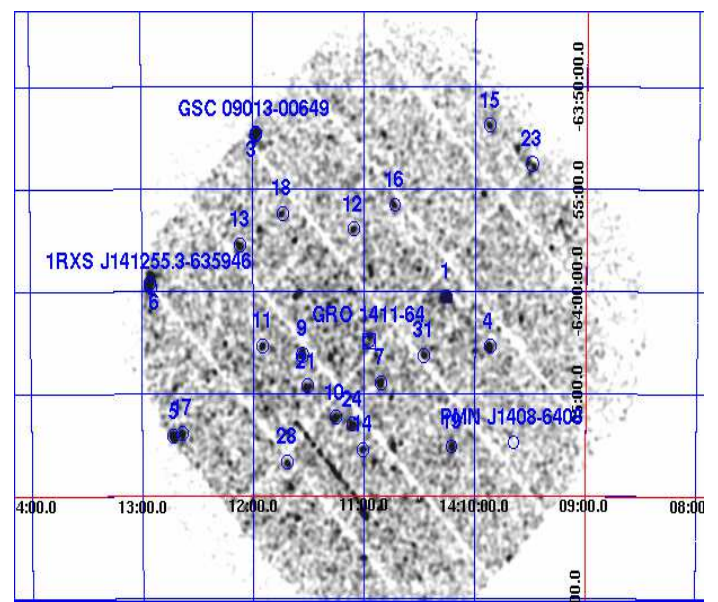

Fig. 5 XMM-Newton EPIC image (combining all 3 cameras), where detected sources and previously catalogued sources have been labeled. The centroid of the COMPTEL source GRO 1411-64 is marked with a square box, the error contour being larger than the image itself. See details in Torres et al. (2006).

moval of background flares, a total of 15.8, 15.8 and 14.6 ks of good data survived for MOS1, MOS2 and pn respectively.

\section{Results}

\subsection{Hard X-rays}

No hint of signal was found for new hard X-ray sources within the location uncertainty of GRO J1411-64 from individual scws of the INTEGRAL instruments. To improve the statistics, mosaic maps were obtained for IBIS/ ISGRI and JEMX by combining all data. The images of IBIS/ ISGRI were produced in the energies 20-100 $\mathrm{keV}$, see Fig. 1 for the map in the $20-40 \mathrm{keV}$ band as an example. The circle holds the $4-\sigma$ error region of GRO J1411-64 obtained by COMPTEL during its flare in 1995 (Zhang et al. 2002). From the possible counterparts of GRO J1411-64 discussed in Zhang et al.'s paper (2002), only the Circinus Galaxy shows up in this error region as seen by INTEGRAL. The most significant detection of Circinus Galaxy is in the energies $20-40 \mathrm{keV}$, at a confidence level of $38 \sigma$. The mosaic map of JEMX shows no significant source feature is visible from within the 4- $\sigma$ error region. For SPI, the Circinus Galaxy is at the $6 \sigma$ level in the $20-40 \mathrm{keV}$ range, and it is the only source detected within the location of GRO J1411-64, the region of our search.

The light curve for the Circinus Galaxy, detected mainly by IBIS/ISGRI, is rather constant. The Circinus Galaxy was investigated by Soldi et al. (2005). Models of cutoffpl plus wabs in XSPEC can fit the data well, with a reduced $\chi^{2}$ of 1.1 ( 7 dof). The resulting parameters are consistent with those in Soldi et al.

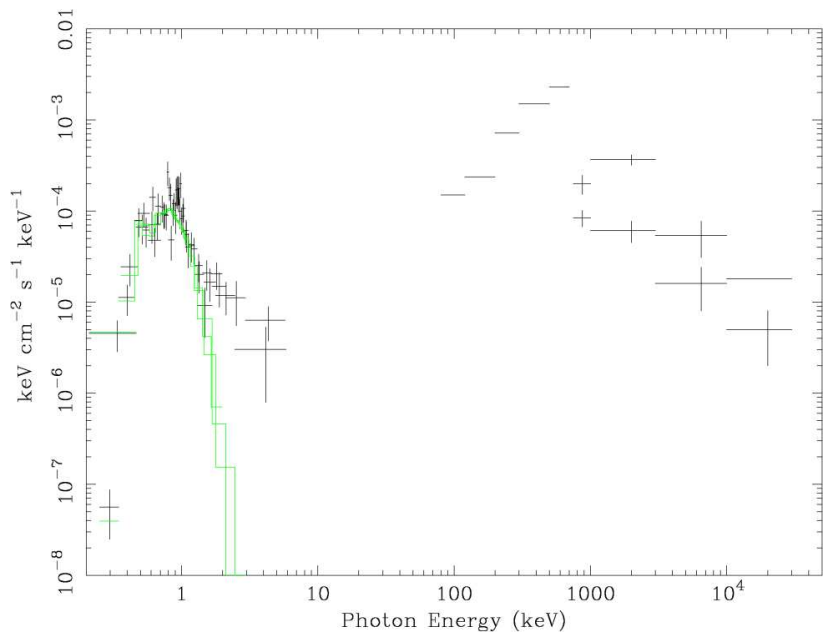

Fig. 6 XMM-Newton unfolded spectrum of the X-ray source XMMU J141255.6-635932. The model shown is only the thermal component in the X-ray spectrum. The COMPTEL detections and the INTEGRAL upper limits are also shown at high energies, with horizontal bars denoting $2 \sigma$ upper limits.

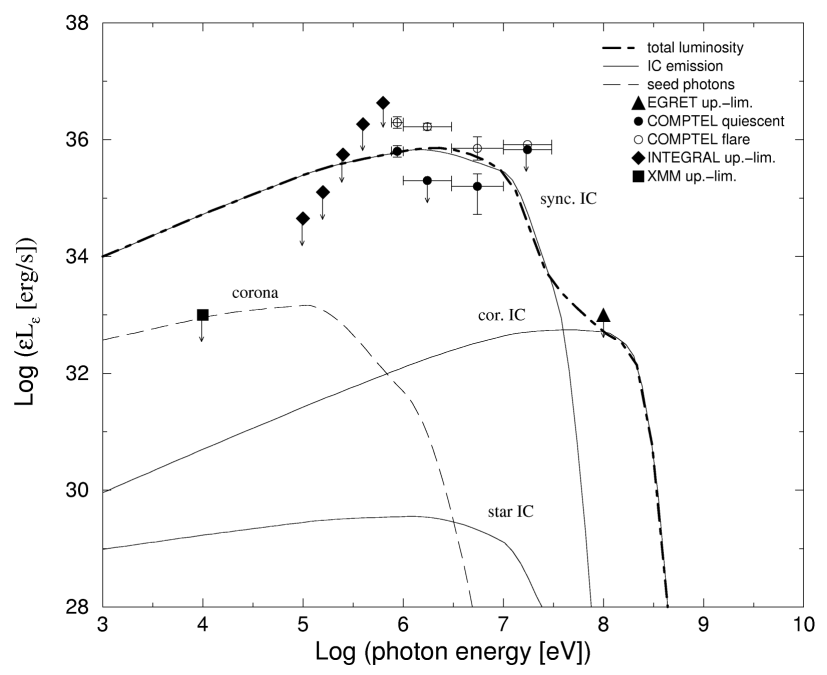

Fig. 7 A microquasar model on the light of observational constraints. See Torres et al. (2006) for details.

GRO J1411-64 shows likely persistent emission in 0.75-1 MeV band during its low state (Fig. 2), where the source was detected at $\sim 4 \sigma$ by COMPTEL (Fig.3). The corresponding spectrum of the low state can be represented by a power law shape with spectral index $2.5_{-0.4}^{+0.6}$ (see Fig. 4). Circinus Galaxy can be safely ruled out as the counterpart due to its spectral extrapolation well below the ones at MeV energies. The ISGRI/SPI upper limits combined to spectra of both flare/low states shows the existence of a maximum in the power output at hard X-rays, which might remind us to consider the microblazar as the possible source nature. 


\subsection{Soft X-rays}

A total of 31 X-ray sources were formally detected by the SAS source detection algorithm in the EPIC data. Nine of these were excluded due to detector defects and other artifacts, in a careful inspection. The resulting 22 reliable and statistically significant sources (likelihood $>10)$ are shown in Fig. 5. Among them, the unfolded spectrum (largely independent of the model fitted) for XMMU J141255.6-635932, along with the best fit model, the COMPTEL detections and the INTEGRAL upper limits is plotted in Fig.6. The hard excess exhibited by the XMM-Newton data is apparent in that figure, and might be suggestive of a large Compton bump that would peak in the several $\sim 100 \mathrm{keV}$ region, fitting well with the COMPTEL detections. However, the fact that the XMM-Newton image does not cover the full COMPTEL source location and the non-detection by INTEGRAL of any reliable counterpart, would make the assumption that XMMU J141255.6-635932 is the counterpart to GRO J1411-64, although spectrally consistent, only tentative and risky.

Table 1 Parameter values for GRO J1411-64. At the top of table, parameter values for a typical microquasar system and jet geometry are given (Bosch-Ramon et al. 2006). We have considered different values within the range open for the free parameters finding that it is not possible to obtain a simple microquasar model that could fit the SED. In particular, in Fig. 7 we show a test case with the free parameters fixed to the values presented in this table, at the bottom. We note that, since the computed SED in Fig. 7 is dominated in the gamma-ray band by SSC emission, the model results would also apply for a low mass microquasar.

\begin{tabular}{cl}
\hline Parameter & values \\
\hline Stellar bolometric luminosity [erg s$\left.{ }^{-1}\right]$ & $10^{38}$ \\
Apex dis. to the comp. obj. [cm] & $5 \times 10^{7}$ \\
Initial jet radius [cm] & $5 \times 10^{6}$ \\
Orbital radius [cm] & $3 \times 10^{12}$ \\
Jiewing angle to the axis of the jet [ $\left.{ }^{\circ}\right]$ & 45 \\
Jet Lorentz factor & 1.2 \\
\hline \hline Jet leptonic kinetic luminosity [erg s $\left.{ }^{-1}\right]$ & $3 \times 10^{35}$ \\
Maximum electron Lorentz factor (jet frame) & $5 \times 10^{2}$ \\
Maximum magnetic field [G] & 8000 \\
Electron power-law index & 1.5 \\
Total corona luminosity [erg s & $\left.3 \times 10^{-1}\right]$ \\
\hline
\end{tabular}

\section{Conclusion and Summary}

The observations, subsequent analysis and theoretical investigations pursued shed light upon the nature of GRO J1411-64. The combined INTEGRAL, XMM-Newton and COMPTEL observations reveal no obvious counterpart at high energies (hard X-rays and gamma-rays). Nevertheless, the unique peak of the power output at these energies resembles the SED seen in microquasars, and suggests at work by a similar scenario. However, an analysis using a microquasar model consisting on a magnetized conical jet filled with relativistic electrons which radiate through synchrotron and inverse Compton scattering with star, disk, corona and synchrotron photons shows that it is hard to comply with all observational constrains (Fig. 7). The best fit parameters see Table 1. This fact and the non-detection at hard X-rays introduce an a-posteriori question mark upon the physical reality of this source. See more details in Torres et al. (2006). GLAST observations would help improving the location of the $\mathrm{MeV}$ source if radiation at higher energies is not completely suppressed, and would open the door for more efficient multiwavelength searches of the counterpart. However, it is true that the nature of this COMPTEL source might not be constrained further if this detection was a one-time only transient phenomena. GLAST will only be able to help if a candidate counterpart is caught in the act (flaring/quiescent state of an AGN or a more rare galactic object). Having at hand GLAST observations, in any case, will make our currently reported investigation to naturally fit into the testing of any hypothesis on the nature of GRO J141164.

Acknowledgements We thank Dr. M.T. Ceballos for her help with the XMM-Newton data. DFT has been supported by Ministerio de Educación y Ciencia (Spain) under grant AYA-2006-0530, and the Guggenheim Foundation. S. Zhang was subsidized by the Special Funds for Major State Basic Research Projects and by the National Natural Science Foundation of China. XB and AC were financially supported for this research by the Ministerio de Educación y Ciencia (Spain), under project ESP2003-00812. VB-R and JMP have been supported by Ministerio de Educación y Ciencia (Spain) under grant AYA-2004-07171-C02-01, as well as additional support from the European Regional Development Fund (ERDF/FEDER). VB-R has been additionally supported by the DGI of the Ministerio de (Spain) under the fellowship BES-2002-2699. GER was supported by grants PIP 5375 y PICT 03-13291.

\section{References}

1. Bosch-Ramon, V., Paredes, J.M., , Romero, G.E. \& Torres, D.F., 2006, A\&A 446, 1081

2. Soldi S., Beckmann V., et al., 2005, A\&A 444, 431

3. Torres D.F., Zhang S.,et al., 2006, A\&A, accepted, see also astro-ph/0606470

4. Zhang S., Collmar W., \& Schönfelder V., 2002, A\&A 396, 923 